\title{
ANOMALY CANCELLATION IN TEN DIMENSIONS AND BEYOND
}

A.N. Schellekens

CERN - - Geneva

\section{ABSTRACT}

\begin{abstract}
A new mechanism to turn Green-Schwaxz gauge anomaly cancellation into complete gravitational anomaly cancellation is discussed. It can be used to derive the type IIB cancellations in $d=10$ and $d=18$ without explicit calculations and also to derive new complete cancellations involving two-form spinors in $d=10$ and $d=14$. We also obtain new Green-Schwarz cancellations for $E_{7}$ in $d=14$ and $S 0(d-2)$ for arbitrary even d. Some aspects of compactification are also discussed.
\end{abstract}

CERN-TH. $4412 / 86$

March 1986 
The revolutionary developments in string theory were initiated by three spectacular examples of higher dimensional anomaly cancellation: the one found by Alvarez-Gaume and Witten ${ }^{1)}$ for the field theory limit of the type IIB superstring, and the Green-Schwarz mechanism for $S O(32)^{2)}$ and $E_{8} \times E_{8}{ }^{3)}$. In a recent paper $^{4)}$, Thierry-Mieg has discovered three even more spectacular cases of anomaly cancellation in $\mathrm{d}=18$ and $\mathrm{d}=26$. Although no corresponding string theory has yet been constructed, definitive ideas about its spectrum exist ${ }^{4)-6)}$. Even without having a string theory, the field theory anomaly cancellation is a rigorous fact, which hints at the existence of more spectacular examples of anomaly cancellation. Well-known mechanisms for obtaining new anomaly-free theories from existing ones are breaking the gauge group to a subgroup, and compactification. We will explore some consequences of these mechanisms in this paper, but our main purpose is to introduce a new mechanism, which produces new anomaly-free theories from existing ones by transforming gauge degrees of freedom into spin degrees of freedom. We will use this to give a straightforward derivation, requiring no explicit calculations, of the type IIB anomaly cancellations in $\mathrm{d}=10$ and $\mathrm{d}=18$. Furthermore, new gravitational anomaly cancellations can be obtained in $d=10$ and $d=14$ (as well as $d=6$ ). This does not disagree with existing results, since these cancellations involve two-form spinors (spinors with two antisymmetric vector indices), and hence are not obviously consistent by conventional standards. We will leave it as an open question whether the potential inconsistencies can be circumvented.

Let us start with compactifications of the $d=18 \mathrm{E}_{8}$ gauge theory ${ }^{4}$ ). Because the consistency condition (i.e., $\operatorname{TrF}^{2}-30 \operatorname{Tr} \mathrm{R}^{2}$ integrated over any closed four-manifold vanishes) is the the same as for $d=10$, many of the results obtained for example in Refs. 7)-10) are immediately applicable. For example, compactifications on spheres give chiral $s 0(2 n-2)$ theories in $d=2 n$, which should be anomaly free. It is well-known that anomaly-free chiral $\operatorname{so}(2 n+6+4 k)$ $(k>0)$ theories exist in $\left.d=2 n^{11}\right)$. Their existence is due to the fact that the on1y index ${ }^{*}$ ) of $S O(2 N)$ which is sensitive to the chirality of a representation is of order $N$, which is too high to affect the anomaly. This index becomes precisely the leading anomaly for $s 0(2 n+2)$ in $d=2 n$. For $s 0(2 n-2)$ the chiral index appears only in subleading terms, and the leading anomaly vanishes again, which makes a Green-Schwarz cancellation possible. If $n=$ even, the expression for the *) will make extensive use of properties of higher indices of Lie algebra
representations, first discussed by okubo and Pateral 2 . Many other useful results may be found in Refs. 10) and 13). 
Atiyah-singer index from which the anomaly can be derived is ${ }^{*} 14$ )

$$
\hat{A}(R) C h(F, r)=\frac{1}{(n+1) \cdot}\left(\frac{i}{2 \pi}\right)^{n+1} \pi_{r} F_{r}^{n+1}+\left(\frac{i}{2 \pi}\right)_{12(n+1)+}^{n} \frac{1}{(4 \pi)^{2}} T_{r} F_{r}^{n+1} \pi_{r} R^{2}
$$

The following trace identity holds for the trace of $n+1$ generators in $\operatorname{so}(2 n-2)(n$ even) (and also for $\operatorname{TrF}^{7}$ in $\mathrm{E}_{6}$ )

$$
\pi F_{r}^{n+1}=\frac{1}{2} \frac{n(n+1)}{(n-1)(2 n-1)} / \frac{d(A)}{d(n)}-\frac{n-1}{12} \frac{T_{2}(A)}{T_{2}(r)} / \pi_{r} F_{r}^{n-1} F^{2}
$$

where $d$ and $I_{2}$ denote the dimension and the second index; $r$ can be any irreducible representation, but the expression vanishes unless $r$ is chiral. Substituting (2) into (1) and converting $\operatorname{TrF}_{r}^{2}$ to a trace over the vector representation we get

$$
\left.\hat{A}(R) C h(F, r)=\left(C_{\mu} \pi_{r} F^{2}-\pi R_{v}^{2}\right)\left(\frac{(i}{2 \pi}\right)^{n+1} \frac{i}{48(n-1) \cdot}, \pi F_{r}^{n-1}\right)
$$

with

$$
\left.C_{\mu}=\frac{12}{(n-1)(2 n-1)} / C_{2}(n)-\frac{n-1}{12} C_{2}(A)\right)
$$

where $C_{2}(r)$ is the quadratic Casimir eigenvalue of the representation $r$. Using a formula for $C_{2}$ derived in Ref. 15), we $c$ an show that $c_{r}>1$ and $c_{r}=1$ if and only if $r$ is a fundamental spinor. The case $n=$ odd is very similar. One considers a left-handed spinor in a chiral representation $r$ of $\operatorname{so}(2 n-2)$ and a right-handed one in the chiral conjugate representation $r^{\prime}$. It is convenient to denote this representation as $r-r^{\prime}$. All symmetric traces of less than $n-1$ generators vanish for $r-r^{\prime}$. For the trace of $n+1$ generators (2) holds, with $\operatorname{TrF}_{r}^{n \pm 1}$ replaced by $\operatorname{TrF}_{r-r}^{n \pm 1}$, but no change in $\operatorname{TrF}_{r}^{2}\left(=\operatorname{TrF}_{r}^{2}\right.$ ). The rest of the discussion is identical.

*) The subscript on $F$ and $R$ indicates over which representation the trace is to be taken. The extra argument in the Chern character has the same purpose. Representations will be indicated by Young tableaux or dimensions; furthermore, the abbreviations $A(d j o i n t) ~ V(e c t o r)$ and $S$ (pinor) are used; $S$ ' is the chiral conjugate spinor. 
For theories with a Green-Schwarz anomaly cancellation, the Green-Schwarz factor may in general (for gauge group $G$ in $d$ dimensions) be written as

$$
C \pi F_{r}^{2}-\frac{I_{2}(r, C)}{I_{l}(r, s 0(a))} \pi R_{r}^{2}
$$

In compactification, the integral of this factor over any closed four-dimensional submanifold must vanish ${ }^{7}$ ). We will call a GS-factor regular if $\mathrm{C}=1$. In that case, one can satisfy the consistency condition by means of an embedding of index 1 of the spin connection into the gauge group. If $C=1 / k, k$ integer, the same can be accomplished by an embedding of index $k$ (if such an embedding exists). if C > 1, spin connection induced compactification is, in general, not possible [apart from exceptional cases, such as spheres, for which (5) vanishes identically]. This is the case for all so(2n-2) theories described above, except when the fermions are in the fundamental spinor representation.

If a theory with a regular GS factor is compactified consistently on some manifold $M$, one obtains either a complete anomaly cancellation, or a regular GS anomaly cancellation. This can be seen by considering spin-connection induced compactification of the original theory on a product manifold $M \times M^{\prime}$. It should be possible to obtain the same result by compactifying the intermediate theory on $M^{\prime}$, and hence its GS factor must be regular. Indeed, all sphere compactifications of the $\mathrm{d}=18$ theory (which has a regular GS factor) yield chiral $\operatorname{so}(2 n-2)$ theories with fermions in fundamental spinor representations.

Another example is compactification of the $\mathrm{d}=18 \mathrm{E}_{8}$ theory on a six-dimensional Calabi-Yau manifold ${ }^{9}$ ), which yields chiral fermions in the 27 of $E_{6}$. In $\mathrm{d}=4$ such a theory is completely anomaly free, but in $\mathrm{d}=12$ it is only GS anomaly free, because $E_{6}$ has a fifth index. The GS factor is $\operatorname{TrF}_{27}^{2}-3 \operatorname{Tr} R_{V}^{2}$, which is regular.

A less trivial case is compactification on $k_{3}$ to $d=14$. The relevant formulas were given in Ref. 10), and we can immediately write down the spectrum in terms of the Euler characteristic $\chi$ and Hirzebruch signature $\tau$ of $k_{3}$. The $\mathrm{SU}(2)$ holonomy group breaks $\mathrm{E}_{8}$ down to $\mathrm{E}_{7}$, and in addition to $(1 / 16) \tau$ gravitinos and $(1 / 16) \tau$ Weyl fermions with opposite chirality, we get Weyl fermions in the $E_{7}$ representation

$$
\frac{1}{16} \tau(133)-\frac{1}{4}(x+2)(56)+\left(\frac{3}{2} x+\frac{29}{16} \tau\right)(1)
$$


Obviously $\tau$ must be a multiple of 16 . The $8^{\text {th }}$ indices of the (133) and (56) are 10 and 1 respectively, and since they contribute to the leading anomaly, they must cancel. This leads to the condition $\chi=3 / 2 \tau$, so that $\chi$ must be a multiple of 24. Remarkably, $\chi=24$ and $\tau=16$ are precisely the correct values for $k_{3}$ [see also Ref. 10) for a similar result]. For $\chi=24$ and $\tau=16$, the total number of Weyl-spinors in (6) is 492, so that what we have found is a gauged version of Thierry-Mieg's $\mathrm{d}=14 \mathrm{gravitational}$ anomaly cancellation ${ }^{4}$. It would be interesting to know if larger gauge groups can be realized on these fermions. One may check the anomaly cancellation by using the $E_{7}$ trace identities

$$
\begin{aligned}
& T_{r} F^{8}=-\frac{1}{36} \pi F_{r}^{2} \pi F_{r}^{6}+\frac{5}{7^{3} 3^{6} 2^{8}}\left(\pi F_{\mu}^{2}\right)^{4} \\
& T_{r} F_{r}=-\frac{1}{196}\left(\pi F^{2}\right)^{2}
\end{aligned}
$$

where $x$ is the representation (133) - 10(56). The Green-Schwarz factor is $\operatorname{TrF}^{2}+$ $42 \operatorname{Tr} R^{2}$, and is regular because $I_{2}(r)=-84^{*}$ ).

We are now ready to discuss the main point of this paper. Consider first the ten-dimensional type IIB superstring. Its field theory limit consists of two left-handed gravitinos $\psi_{L}^{a}$, two right-handed Majorana-Weyl spinors $\psi_{R}$ and a selfdual rank 4 antisymetric tensor $\phi$. Their Atiyah-Singer indices are ${ }^{14)}$

$$
\begin{aligned}
& \left.A\left(\psi_{L}^{a}\right)=\hat{A}(R) / C h(R, V)-1\right) \\
& A\left(\psi_{R}\right)=-\hat{A}(R) \\
& A(\phi)=-\hat{A}(R) \operatorname{Ch}(R, S)
\end{aligned}
$$

where $\mathrm{V}$ and $\mathrm{S}$ denote the (10) and (16) of So(10). To show that the last term has the correct normalization, one may re-express the trace in the Chern character in terms of a trace over the vector representation, and obtain the Hirzebruch signature polynomial with the correct factor $1 / 414$ ). To show that the theory is anomaly free, we decompose all $S O(10)$ generators in (8) to so(8) subalgebra generators. In general, anomaly cancellation for subalgebras is necessary, but not sufficient for anomaly cancellation in the full theory. However, so(8) is a

*) $I_{2}$ is normalized as in Ref. 16). Notice that the GS-factors in $d=10,14$ and 18 can all be written as $\operatorname{Tr} R^{2}+B_{n} \operatorname{Tr} F^{2}$, where $B_{n}(d=2 n+2)$ are the Bernoulli numbers $\left(B_{4}=-1 / 30, B_{6}=1 / 42, B_{8}{ }^{n}=-1 / 30\right)$. 
large enough subalgebra because it has a sixth index. The relevant so(10) $\supset$ So(8) decompositions are

$$
\begin{aligned}
& V=(10)=(8 v)+2(1) \\
& S^{\prime}=(16)=\left(8_{s}\right)+\left(8_{c}\right)
\end{aligned}
$$

Applying these decompositions to the Chern characters in (8) (the Dirac genus does not change), we get the following expression for the total anomaly

$$
\begin{aligned}
A_{\text {tot }} & =2 A\left(\psi_{t}^{4}\right)+2 A\left(\psi_{R}\right)+A(\phi) \\
& =\hat{A}(R)\left[2 C h\left(R, B_{r}\right)-C h\left(R, B_{s}\right)-C h\left(R, B_{c}\right)\right)
\end{aligned}
$$

From the previous discussion we have learned that in 10 dimensions, there exists a Green-Schwarz anomaly free So(8) gauge theory, so that

$$
\hat{A}(R)\left(C h\left(F, B_{s}\right)-C h\left(F, B_{0}\right)\right) \propto\left(\pi F^{2}-\pi R^{2}\right)
$$

Using so(8) triality we find therefore

$$
\left.\hat{A}(R) / 2 C h\left(F, B_{2}\right)-C h\left(F, B_{s}\right)-C h\left(F, B_{c}\right)\right) \propto\left(T F^{2}-T_{1} R^{2}\right)
$$

The last step is obvious: to go from (11) to (9), one must replace F by $R$; the Green-Schwarz factor vanishes, and the gravitational anomalies cancel completely.

Consider now the type IIB theory in $\mathrm{d}=18$. In the light-cone frame its so(16) field content is

$$
[(16)+(120)+(128)] 0[(16)+(120)+(128)]
$$

This yields two two-form spinors $\psi^{[a b]}$ plus a self-dual tensor; the other fermions appear in non-chiral combinations. The anomaly for a two-form spinor in d dimensions is ${ }^{4)}$

$$
A\left(\psi^{[a b]}\right)=\hat{A}(R) / C h(R, B)-C h(R, \nabla)+1 J_{\text {so(d) }}
$$

(assuming that a coupling to gravity can be constructed which produces the anomaly dicated by the index theorem). Decomposing this to $s o(d-2)$ we get 


$$
A\left(\psi^{[067}\right)=\hat{A}(R) / C h(R, \theta)+C h(R, \square) /
$$

It is, of course, no coincidence that this is the Chern character of the $s 0(d-2)$ representation from which the fermions originated. Multiplying by two and adding the contribution of the self-dual tensor, reduced to so(16), we get

$$
\begin{aligned}
A_{\text {tot }}=\hat{A}(R) & +2 \operatorname{Ch}(R, \theta)+2 \operatorname{Ch}(R, 0) \\
& +\operatorname{Ch}(R, 128)+\operatorname{Ch}\left(R, 128^{\prime}\right) \int_{\text {so(R) }}
\end{aligned}
$$

This $c$ an be related to the $E_{8}$ gauge anomaly cancellation in $d=18$, which reads

$$
\left.A_{\text {gange }}=\hat{A}(R) \operatorname{Ch}(R, a)+C h(F, 248)\right) \propto\left(\pi F_{28}^{2}-30 \pi R_{\nu}^{2}\right)
$$

Breaking $\mathrm{E}_{8}$ down to $\mathrm{SO}(16)$ we get

$$
\begin{gathered}
\text { Agange }=\dot{A}(R)\left|C h(R, a)+C h(F, 120)+C h\left(F_{1}, 28\right)\right| \\
\propto\left(\pi F_{16}^{2}-\pi R_{v}^{2}\right)
\end{gathered}
$$

Adding the same expression with (128') instead of (128), and replacing $F$ by $R$ we find that (14) vanishes. Notice however that in $d=10$ and $d=18$ we had to use the opposite sign for the self-dual tensor anomaly [compare (8) and (14)], a fact also observed in Ref. 4). On the basis of Ref. 14) we have to conclude that the sign used in ten dimensions is correct, and the one used in $d=18$ is wrong. Therefore, there is certainly a spectacular gravitational anomaly cancellation in 18 dimensions, but it seems to correspond to the field theory limit of a type IIB "string" only after the chirality of the self-dual tensor is flipped. This is rather remarkable in view of the fact that we have derived the anomaly cancellation from one of the two constructions of the theory, namely the identification of part of the gauge group with the transverse Lorentz group. [There is a similar construction in $\left.\mathrm{d}=10^{17)}, 5\right)$, but it involves a truncation which makes it imposible to derive an anomaly cancellation from it.]

This transformation can easily be generalized, with one minor difference. In both cases discussed above, some gauge bosons transformed into fermions. In general, we cannot allow that, because bosons do not contribute to the anomaly in the original theory, and are therefore uncontrollable. (The reason it worked previously has to do with the closed string origin of these theories.) Instead, we will simply discard the bosons, and apply the transformation only to the fermions. Consider a Green-Schwarz anomaly-free theory in $d=4 k+2$ dimensions, 


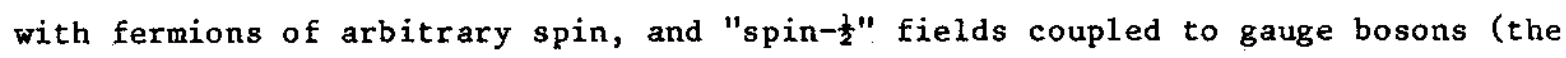
generalization to higher spin fields coupled to gauge bosons is straightforward). The anomaly of this theory is given by

$$
\hat{A}(R) C h(F, r)+\text { gauge singlet contributions }
$$

where $r$ is the representation of the fermions for the gauge group $G$. Now consider a subgroup $s 0(d-2) \times G^{\prime} G$, so that $r$ decomposes as $\Sigma_{i}\left(r_{i}, r_{i}^{\prime}\right)$. The corresponding decomposition of the Chern character is

$$
\operatorname{Ch}\left(E_{1} n\right)=\sum_{i} \operatorname{Ch}\left(E_{1}, r_{i}\right) \operatorname{Ch}\left(F_{2}, r_{i}^{\prime}\right)
$$

where $F_{1}$ and $F_{2}$ are valued in $S O(d-2)$ and $G$ respectively. Now we write each $r_{i}$ as the sum of $\mathrm{so}(\mathrm{d})$ representations. This is in general not possible, but we may allow minus signs because they can be interpreted by flipping the chirality of the fermion. The uniqueness of the procedure - apart from addition of conjugate pairs - is guaranteed by imposing all index sum rules ${ }^{12)}$ on the result. There is a problem if $r_{i}$ is a spinor, since it needs its conjugate to fit into so(d). This problem arises if $\operatorname{so}(d-2) \subset G$ is not self-conjugate [e.g., $\left.\operatorname{so}(16) \subset E_{8}\right]$, and can always be solved by adding the theory constructed from the conjugate embedding. (In general, this does not destroy the non-trivial chirality of the theory.) If $r_{i}=\Sigma_{j} \varepsilon_{i j} r_{i j}, \varepsilon_{i j}= \pm 1, r_{i j}$ is an so(d) representation, we can write (18) as

$$
C h\left(F_{1}\right)=\sum_{i j} \varepsilon_{i j} \operatorname{Ch}\left(F_{1}, r_{i j}\right) \operatorname{Ch}\left(F_{e}, P_{i}^{\prime}\right)
$$

Now we replace $F_{1}$ by $R$ and interpret the result. To relate $C h\left(R, r_{i j}\right)$ to the anomaly of a physical field, we have to add ghosts. Ghosts would contribute to the anomaly, but we can always keep the anomaly cancellation we already have by cancelling every ghost against new physical particles of the same spin. This procedure can be carried out iteratively, starting with the largest representations. It seems very plausible that a much simpler way to obtain the correct field content is to multiply the $\operatorname{so}(d-2)$ representations $r_{i}$ with an $\operatorname{So}(d-2)$ spinor, with an additional factor $\frac{1}{2}$ for the multiplicity of the self-dual tensor. This makes sense because the so(d-2) representations give the proper on-shell count of physical states which is precisely what the ghosts are supposed to achieve. It also agrees with all known examples. These ghost counting arguments are in fact the main reason for using the subgroup so(d-2). One could also use so(d) itself, but this requires a bigger gauge group. With some care one can even get meaningful results with groups smaller than $S(d-2)$ in certain cases. 
There are many obvious applications of these ideas. The $\operatorname{so}(2 n-2)$ theories in $d=2 n$ discussed earlier seem ideally suited, but unfortunately they lead to non-chiral theories, except for $d=10$ where $s 0(8)$ triality can be used, as explained earlier. Other obvious candidates are the $\mathrm{s} O(32)$ and $\mathrm{E}_{8} \times \mathrm{E}_{8}$ theories $\left.{ }^{*}\right)$ in $d=10$ which yield the following chiral theories

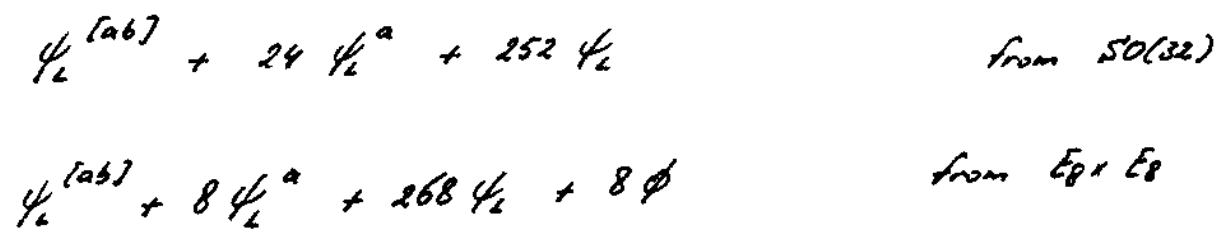

The difference between these two theories is eight times the anomaly free combination of the type IIB superstring, so that there is one new, independent anomaly cancellation among different spins. This is equally remarkable as the type IIB cancellation: given the fact that the anomalies of $\phi_{L}^{a}, \psi_{L}$ and $\phi$ are dependent, one would have expected to need two additional spins to get a new anomaly cancellation. In other words, the anomalies of the four fields appearing in (20) depend on just two linear combinations of $\operatorname{Tr} R^{6}, \operatorname{Tr} R^{4} \operatorname{Tr} R^{2}$ and $\left(\operatorname{Tr} R^{2}\right)^{\left.3^{* *}\right)}$. The rather large multiplicities of the fields in (20) have a natural interpretation in terms of the $S O(24)$ and $\mathrm{E}_{8}$ gauge interactions left unbroken in the procedure [the second $S O(8)$ group in $S O(8)$ is broken because the multiplicity of $\phi$ was obtained as $\frac{1}{2}\left(8_{c}+8_{s}\right)$; furthermore it is not known how to couple gauge fields to self-dual tensors]. Unlike the gravitational anomalies, the gauge anomalies do not cancel completely, but they can be removed by the Green-Schwarz mechanism. Because the only condition for a Green-Schwarz cancellation of the gauge anomaly is that the total sixth index vanishes, there is in fact an enormous amount of additional possibilities, especially since non-chiral combinations like $\psi_{L}+\psi_{R}$ may be added.

Using the $S 0(12)$ subgroup of $E_{7}$ we can construct a new anomaly free multiplet in $d=14$, namely

*) The $S O(16) \times S O(16)$ anomaly cancellation found in Ref. 18) can be obtained as the difference of the $\mathrm{E}_{8} \times \mathrm{E}_{8}$ and $\mathrm{So}(32)$ theories, both broken to $\operatorname{so}(16) \times$ so(16). It is therefore not an independent cancellation.

**) This is not the case for fields of arbitrary spin. For example, the anomalies of three-form spinors or symmetric tensor-spinors do depend on the third linear combination of the curvature invariants. 


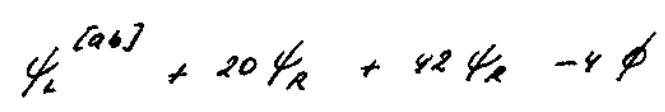

This multiplet can also be obtained by compactification of the 18-dimensional type IIB on $\mathrm{K}_{3}$. Similarly, linear combinations of the multiplets in (20) emerge by compactification on eight-dimensional manifolds, but without the $\operatorname{so}(24)$ gauge group.

There are certainly more possibilities, but a relatively simple picture seems to be emerging in which all simple anomaly free multiplets originate from only a handful of basic ones. Simple gravitational anomaly cancellations seem to exist only for $\mathrm{d}=2,6^{19)}, 10,14,18$ and 26 . The existence of a cancellation for $\mathrm{d}=14$ is adequately explained by $\mathrm{K}_{3}$ compactification of the $d=18$ theories. The absence of a simple cancellation for $d=22$ seems at first paradoxical because an anomaly-free theory exists in $d=26$. This theory is however only GS anomaly free, and the GS factor is $\operatorname{Tr} R^{2}$. If the theory is compactified on any four-dimensional manifold, the consistency condition $\int \operatorname{Tr} R^{2}=0$ implies that the Atiyah-singer index must vanish, so that it is impossible to obtain chiral fermions in $\mathrm{d}=22$.

\section{ACKNOWLEDGEMENTS}

I am grateful to J. Thierry-Mieg for discussions and explanations of his work, and to L. Alvarez-Gaume for a discussion regarding the sign of self-dual tensor anomalies. 


\section{REFERENCES}

1) L. Alvarez-Gaume and E. Witten, Nuc1. Phys. B234 (1983) 269.

2) M.B. Green and J. Schwarz, Phys. Lett. 149B (1984) 117.

3) J. Thierry-Mieg, Phys. Lett. 1568 (1985) 199.

4) J. Thierry-Mieg, Meudon preprint FGAR-86-1 (1986), to appear in Phys. Lett. B.

5) F. Englert, H. Nicolai and A.N. Schellekens, CERN preprint TH.4360/86 (1986).

6) W. Nahm, Bonn preprint Bonn-HE-85-37 (1985).

7) E. Witten, Phys. Lett. 149B (1984) 351.

8) M.B. Green, J.H. Schwarz and P.C. West, Nuc1. Phys. B254 (1985) 327.

9) P. Candelas, G.T. Horowitz, A. Stromingex and E. Witten, Nucl. Phys. B258 (1985) 46.

10) K. Pilch and A.N. Schellekens, Nucl. Phys. B259 (1985) 637.

11) E. Witten, in Proc. of the 1983 Shelter Island Conf. ed. N. Khuri et al., (MIT Press, 1985).

12) S. Okubo and J. Patera, J. Math. Phys. 24 (1983) 2722; 25 (1984) 219.

13) A.N. Schellekens, Nucl. Phys. B250 (1985) 252.

14) L. Alvarez-Gaume and P. Ginsparg, Annals of Phys. 161 (1985) 423.

0. Alvarez, I. Singer and B. Zumino, Comm. Math. Phys. 96 (1984) 409.

15) K. Pilch and A.N. Schellekens, J. Math. Phys. 25 (1984) 3455.

16) W. McKay and J. Patera, Tables of dimension indices and branching rules for representations of simple Lie algebras, (Dekker, New York, 1981).

17) A. Casher, F. Englert, H. Nicolai and A. Taormina, Phys. Lett. 162B (1985) 121 .

18) L. Dixon and J. Harvey, Princeton preprint (1986);

L. Alvarez-Gaume, P, Ginsparg, G. Moore and C. Vafa, Harvard preprint HUTP-86/A013 (1986).

19) S. Randjbar-Daemi, Abdus Salam, E. Sezgin and J. Strathdee, Phys. Lett. 151B (1985) 351 . 